\title{
Teaching epidemiology: An overview of strategies and considerations
}

\author{
Md Mahbub Hossain* \\ School of Public Health, Texas A\&M University, TX 77840.
}

\begin{abstract}
Epidemiology is one of the foundational subjects of public health sciences that is widely taught in academic and non-academic settings. Considering a growing interest in epidemiology in diverse learners, the scholarly community should learn and use effective strategies for teaching epidemiologic concepts and methods. This article reviewed the existing literature and explored useful approaches for teaching epidemiology such as determining the scope of teaching epidemiology for learners with varying learning needs and interests, using conceptual frameworks, allocating academic resources, using realistic epidemiologic studies, deploying innovative educational technologies, strengthening mentoring capacities, using problemsolving practices, promoting experiential and team-based learning, developing academiapractice partnerships, and facilitating continued epidemiologic education. These strategies should be contextualized based on the academic and practical needs of the learners in different contexts. Moreover, implementation research on epidemiologic education is recommended for better understanding the operational challenges and opportunities for teaching-learning practices. Lastly, inclusive and collaborative approaches should be adopted to ensure epidemiologic education and practice continuum that may improve public health policymaking and outcomes.
\end{abstract}

Keywords: Epidemiology; Education; Medical Education; Public Health Education; Knowledge Development; Educational Research; Pedagogic Strategies.

\footnotetext{
*Correspondence to: Md Mahbub Hossain MBBS MPH FRSPH, School of Public Health, Texas A\&M University, TX 77843. Email: mahbub321@gmail.com
} 


\section{Background}

Epidemiology is recognized as the "basic science of public health" and integrated into academic curricula of undergraduate and graduate programs in medicine, public health, nursing, and a growing number of scholarly disciplines [1, 2]. The increasing interest in epidemiologic education can be attributed to the societal and institutional needs to understand public health sciences [3-5]. In their report titled "Who Will Keep the Public Healthy? Educating Public Health Professionals for the 21st Century," the Institute of Medicine recommended that "all undergraduates should have access to education in public health" [6]. Similar recommendations from leading healthcare organizations encouraged many academic institutions to offer stand-alone courses in epidemiology and epidemiologic modules within public health courses [7-10]. Teaching epidemiology aims to promote scientific thinking among future citizens about health and diseases in communities and populations [10-12]. In that way, epidemiology is becoming useful not only to the scholars and practitioners of science but to educate citizens who wish to learn about and make informed decisions about their health and wellbeing.

The academic and professional fields related to epidemiology have grown substantially over the past few decades, which is evident by the evolving lexicon of epidemiologic terminology and our understanding of distributions and determinants of diseases [9]. Most programs and textbooks in foundational epidemiology emphasize definitions, measures of disease distributions and determinants, critical thinking on disease causation, history of epidemiology and public health, study designs, sources of bias, and relevant quantitative measures $[9,11]$. These concepts shape the basic understanding of epidemiology among the students; however, advanced scholarship on epidemiology may focus on specialized knowledge and skills as included in graduate, doctoral, or post-doctoral education and research.

The current paradigm of epidemiologic education may raise some concerns, including deviance from the broader public health perspective by studying a few risk factors in the context of single or a few health outcomes, poor translation of evidence into public health action, and the use of approaches beyond the scope of public health or social context $[11,13]$. In addition, emerging perspectives on using methods or interpreting epidemiologic findings may inform the development of epidemiologic education [13, 14]. For example, the American Statistical 
Association's clear position against reliance on a simple P-value in the study interpretations is often disregarded in many epidemiologic studies [11,15], which informs the need to revisit how these concepts are taught and practiced in epidemiology programs. Also, epidemiology students should be educated about the precision of estimates, data quality, sources of bias, critical evaluation of the findings, and systematic synthesis of epidemiologic evidence to inform practice $[7,9,13,16]$. The role of epidemiologic education should focus on the emerging needs of health professions and social developments that require epidemiologic perspectives for better public health $[11,17]$.

As learners from diverse educational backgrounds are increasingly joining academic programs in epidemiology, the depth and contents of epidemiologic education should be structured considering the needs of the learners in respective contexts $[11,18,19]$. In addition, many institutions are encouraging undergraduates and high school students to take introductory epidemiology. Such a growing diversity of interests, previous education, prospective coursework, lived experiences, and career trajectories among the learners impose unique challenges for teaching epidemiology $[3,5]$. Therefore, epidemiology educators must acknowledge that there is no "one size fits all" approach for developing and implementing epidemiologic curricula. Moreover, the learning outcomes for epidemiologic education must match the academic and professional competencies that the learners should achieve at the end of their programs.

There are several challenges and concerns for teaching epidemiology that may require the attention of the academic community. First, it is necessary to evaluate strategies for effective epidemiologic education for students with diverse backgrounds and interests. Also, epidemiologic education should contribute to the learners' overall intellectual, social, and human development $[13,20]$. Therefore, connecting the dots between epidemiologic theories and real-life public health and wellbeing. More importantly, there is a need to develop effective educational approaches that engage the learners, educators, and institutions to promote sustainable knowledge development in epidemiology. Despite the growing demand for epidemiologic education across institutions and communities, little is known about how educators and academic programs can effectively address such concerns and improve the quality of teaching-learning practices in this field. This article aimed to offer insights on 
teaching strategies and considerations for academic epidemiology using the available evidence and perspectives that may empower the educators, learners, institutions, and academic communities.

\section{Methods}

A critical narrative review of the literature was conducted from scholarly pieces indexed in Medline, Google Scholar, American Psychological Association (APA) PsycInfo, Academic Search Ultimate, and the Cumulative Index to Nursing and Allied Health Literature (CINAHL) databases. Several keywords such as "teaching epidemiolog*", "epidemiologic education*", "learning epidemiolog*", and "academic epidemiolog*" were used for searching those databases. Further, the titles and abstracts were reviewed to evaluate their relevance with epidemiologic education. Articles appearing to be relevant were selected for full-text assessment, and only those presenting insights on epidemiologic teaching-learning were finally included in this review.

Teaching strategies and considerations may not be reported in empirical studies alone, and many academicians may share their experiences and perspectives in commentaries, editorials, and reviews. For this reason, this article used different types of publications from scholarly journals that offered insights on epidemiologic education irrespective of article type or methods. Specific teaching approaches developed, adopted, or proposed by the academic community were identified from the literature and synthesized narratively in this article.

\section{An overview of strategies and considerations for teaching epidemiology}

\section{Determining the scope of teaching epidemiology}

It is necessary to determine the scope of teaching epidemiology in a specific context depending on various circumstances, such as the level of education, previous coursework, and lived experiences of the learners [17, 19, 21]. Educators and institutions may need to determine the meaning and relevance of epidemiology to the learners. For example, medical students joining a course in epidemiology may know about diseases and related factors in a community or a 
population $[18,22]$. However, students from non-health backgrounds joining the same course may struggle or find it difficult to understand different terminologies used in describing the distribution and determinants of a health condition [4, 14, 23]. Moreover, some students may join an epidemiology course at the beginning of their respective academic programs (e.g., in public health, medicine, nursing etc.), who would use this knowledge in their future coursework but have limited knowledge at the initial stage [9, 24, 25]. Furthermore, the academic needs of introductory epidemiology for undergraduate students are likely to be different than those of masters or doctoral students, who would need a more fundamental level of orientation to epidemiology. As they learn more and join advanced sections of epidemiology, there will be critical concepts (e.g., survival analyses) that would need some previous coursework on basic concepts (e.g., measures of association and causality, study designs etc.) to fully appreciate the same. Such challenges must be acknowledged, and they should inform the framing of course curricula, contents, and resources in respective contexts.

Previously, epidemiology was generally taught to advanced students of health sciences who would primarily work on healthcare domains such as public health workers, researchers, doctors, nurses, allied health scientists, social scientists working on health issues $[6,13,19]$. However, in recent years, the significance of epidemiologic education has been increasingly recognized across different branches of knowledge. Over the past two decades, scholars are emphasizing the need for epidemiologic education in high schools and colleges for educating future citizens about population health and wellbeing [4, 7]. Moreover, non-academic institutions and communities might wish to learn the art and science of epidemiology and public health due to a growing interest in public health since the coronavirus disease (COVID-19) pandemic $[26,27]$. These diverse groups of learners may have different educational needs that should guide the development of educational curricula and content.

Epidemiology educators may need to consider such factors and initiate conversations with fellow learners to fully understand the scope of epidemiology in respective contexts. Inclusive and futuristic development of learning goals and objectives should be encouraged to fit the learners' knowledge needs. Further, all educational stakeholders (e.g., learners, educators, administrators, institutions, communities) must revisit such developments and reframe the scopes of learning epidemiology based on the intellectual and socio-ecological changes. In these 
processes, the educators should evaluate the relevance of suggested educational measures in the context of local and global standards of epidemiology education. In the U.S., for example, most academic programs in public health use the Council on Education for Public Health (CEPH) standards for ensuring the quality of public health education [28, 29]. Similar accrediting institutions may provide additional guidance on expected educational processes and outcomes, which can be useful to determine the scope of teaching epidemiology in respective contexts.

\section{Developing and implementing conceptual or strategic frameworks}

Effective teaching-learning approaches would require a strategic roadmap that may inform what concepts and skills should be learned, how they should be organized and delivered, and how to ensure optimal articulation of different ideas within the broad field of knowledge [30, 31]. For this reason, the role of curricula or syllabi is critical for structured learning programs in epidemiology. However, such materials should be used as parts of multiple strategic tools that can be used for organizing the contents and activities related to epidemiology. If multiple strategic tools or frameworks are used for teaching-learning processes, they should be harmonized for the learners and educators so that they can use the same for accomplishing all learning goals and outcomes in a planned manner.

Many educators use purpose-based frameworks for teaching epidemiology and encouraging the students to engage in broader epidemiologic discourses. Avelino-silva and colleagues suggested the "Big 6" approach [32], which informs the six most common purposes or objectives of epidemiologic investigation as highlighted in Table 1. The use of such questions and frameworks can promote a better understanding of epidemiologic concepts and methods. It should be noted that key epidemiologic concepts are not similarly presented across the introductory textbooks. Keyes and Galea identified key concepts such as basic measures of disease occurrence (as prevalence and incidence, risks, and rates, etc.), an overview of descriptive epidemiology and surveillance, study designs, bias, confounding, and basics of interaction in most textbooks [9].

Despite presenting similar key topics across those books, teaching introductory courses on epidemiology can be problematic due to critical issues such as:i) building a sequence of concepts mostly based on their historical evolution, ii) existence of disciplinary arguments on definitions 
and terminologies, and iii) use of discrete concepts without sufficient overarching and fundamental principles.

Table 1: "Big 6" purposes of epidemiology

\begin{tabular}{|c|c|}
\hline $\begin{array}{l}\text { Purpose, objective, } \\
\text { or goal of } \\
\text { epidemiology }\end{array}$ & Explanations/sample questions \\
\hline Description & $\begin{array}{l}\text { - } \quad \text { How often does health condition "Y" occur? } \\
\text { - } \quad \text { How many people were affected by condition "Y"? } \\
\text { - } \quad \text { What is the trend of occurring "Y" from year "A" to year "B"? }\end{array}$ \\
\hline Causation & $\begin{array}{l}\text { - What is the relationship between "X" and "Y"? } \\
\text { - Does factor "X" cause health condition "Y"? }\end{array}$ \\
\hline Mediation & - How does factor "X" cause health condition "Y"? \\
\hline Attribution & $\begin{array}{l}\text { - How much of problem "Y" can be attributed to factor "X"? } \\
\text { - What proportion of " } Y \text { " can be eliminated if causal "X" is } \\
\text { eliminated? }\end{array}$ \\
\hline Interaction & $\begin{array}{l}\text { - } \quad \text { For which population group does "X" cause "Y"? } \\
\text { - } \quad \text { Under which condition(s) does "X" cause "Y"? }\end{array}$ \\
\hline Prediction & - Do "P", "Q", and "R" predict the occurrence of "Y"? \\
\hline
\end{tabular}

Keyes and Galea highlighted the need for addressing these concerns, recalibrating the existing teaching measures, and developing a more cohesive approach to teaching epidemiology [9]. Specifically, they suggested seven foundational steps of teaching how to conduct an epidemiologic study, as listed in Table 2.

Such frameworks or sets of techniques can be used to facilitate teaching-learning exercises in epidemiology. Furthermore, educators may use a "concept mapping" approach to prospectively prepare for, or retrospectively reflect on, a wide range of epidemiologic concepts, methods, and competencies. According to Berglund [12], a concept map is constructed "using a set of concepts 
(nodes) that are linked with arrows or lines (links). Words and phrases (connective terms) are used to explain relationships between the concepts linked. Different domains can be interconnected by linking concepts in different areas (cross-links). The underlying structure of knowledge is often complex, and consequently, concept maps can be constructed using different topological features."

\section{Table 2: Steps of conducting an epidemiologic study}

\section{Steps}

Description

1 Defining the community/population of interest

2 Conceptualizing and developing measures of health conditions, indicators, and exposures of interest

3 Specifying a sample of the population

$4 \quad$ Estimating measures of association between health indicators and exposures

$5 \quad$ Evaluating causal associations among variables of interest

6 Assessing potential evidence of multiple causes working together

$7 \quad$ Evaluating external validity, the extent to which the results inform similar phenomenon, in other populations

In classrooms and other learning contexts, the educators may engage the learners to think about the epidemiologic concepts of interest that they learned and prepare maps connecting multiple concepts. Rather than developing a single map, the learners may keep editing their maps and compare multiple versions of their own work. This active learning process may enable them to realize the growing interconnectedness between multiple concepts and promote critical thinking, a rare commodity in the modern age.

\section{Allocating resources essential for learning epidemiology}

Despite a growing interest in academic epidemiology, there is little evidence on academic resources available in most institutions that offer courses on epidemiology. For example, using practice labs and external sites for epidemiologic education are some of the materialistic resources needed for teaching-learning exercises. Such resources might be available in institutions with fully developed departments of epidemiology or public health. However, this 
may not be the case for institutions where epidemiology is not a major concentration, or it is taught as a part of another health-related discipline, or in resource-limited contexts. We must recognize the intellectual burden it may produce if the learners cannot avail or access these resources to apply classroom theories in practice.

Institutionalized programs, curricula, or academic arrangements for teaching and learning epidemiology are some of the critical resources that may potentially impact the level of epidemiologic knowledge and practice among scholars and practitioners in those contexts [21, 28]. For example, medical schools may have a provision for teaching preventive medicine without a specific course or section on epidemiology, which may leave the future doctors with limited epidemiologic knowledge. In contrast, universities with schools of medicine and public health may potentially benefit from having dedicated resources on epidemiology that can be accessed by learners from diverse academic disciplines. Such resources within the same institution can be considered as bonding capital (a form of social capital within the same community or context), whereas partnerships with external institutions for learning epidemiology can be termed as bridging capital (a form of social capital between two or more different entities). The use of social capital is widely studied in different fields [33, 34]; however, how such capital can be developed and utilized in academic epidemiology is yet to be explored.

Educators and other academic decision-makers in epidemiology should analyze the strengths and weaknesses of the existing institutional resources and arrangements for epidemiologic education and compare the same with what is needed for optimal teaching-learning outcomes. These challenges can be even more prevalent in resource-constrained contexts that may have a more severe scarcity of academic resources [35]. The academic community should facilitate discussions on how to overcome such scarcities and improve epidemiologic education as part of broader measures for strengthening public health research and practice in those contexts.

\section{Using realistic epidemiologic studies}

Most educational programs on epidemiology focus on concepts and methods, whereas there is a growing need to use real examples and cases to highlight the relevance of those theoretical measures. A study by Moffat and colleagues found that many medical students perceived learning epidemiology as a complicated process because the epidemiologic topics appear to be 
dry, boring, and difficult to understand [21]. However, they acknowledged the importance and relevance of epidemiology as they progressed through the medical course. Therefore, practicefocused and clinically-relevant measures of teaching epidemiology should be encouraged whenever possible.

Epidemiology, as a fundamental science of public health, must attempt to understand health from a socio-ecologic perspective, and study how society is organized, what impacts socioeconomic forces may have on health, and what community actions may change the distribution of health outcomes in populations $[6,10,11,20]$. In most contexts, epidemiology has become a discipline of biomedical sciences that focus on the distribution and determinants of diseases in individuals or communities sharing common exposures or diseases. In such cases, the ecology of human health or the societal context in which health or disease manifests are mostly underexamined, disregarded, or abstracted [36]. To address such limitations of current teaching practices, epidemiology educators may use context-specific case scenarios that facilitate an introduction to core concepts or methods, promote critical reasoning among the learners, and allow them to use epidemiology alongside other branches of knowledge to understand the basis of health problems around themselves [8, 21,37].

\section{Digital media and innovative learning technologies}

Digital tools and technologies are increasingly used for educational purposes in many disciplines, including epidemiology. Both the Association of Schools and Programs of Public Health and the U.S. Public Health Service have recognized online education on public health sciences [11]. A notable example of an online epidemiology resource is the "Principles of Epidemiology in Public Health" by the Centers for Disease Control and Prevention (CDC) [38]. Similar open-access programs on epidemiologic concepts and methods exist thanks to academic and development organizations. However, the actual use of digital resources within academic epidemiology programs is yet to be evaluated. Many schools of public health offer online classes and programs that can be attended and completed without in-person attendance [11, 14, 39]. Also, studies from different disciplines suggest similar or better educational outcomes in online education [40,41]. Moreover, a wide range of tools and resources exist in the digital world that are increasingly adopted in epidemiology education. 
These technological advancements reflect the growing interests of the academic community to develop and implement resources that fit context-specific educational needs in epidemiology. For example, Mobasheri and colleagues evaluated the efficacy of epidemiology learning software using a quasi-experimental study in 101 nursing and midwifery students [42]. The students were divided into two groups: the intervention group members were taught via the software and another group received education as usual. After the semester, the researchers found a significant difference in epidemiology scores between the two groups $(p<0.001)$. Moreover, the intervention group students obtained significantly higher scores compared to the traditionally taught students. Another study by Russell and colleagues described the use of computer-assisted instruction (CAI) as an adjunct teaching-learning strategy in epidemiology [43]. This program was delivered through an interdisciplinary team, and the participating students $(n=106)$ believed the educational content was clearly explained. Moreover, they preferred learning epidemiology through combining classroom learning with CAI rather than traditional format text-based learning and assignments.

Such use of digital tools and technologies is not new in epidemiology education. One of the earliest examples of digital resources used in this field was described in a 1998 article by Dean and colleagues [44]. They discussed the "DoEpi" series of computer exercises and presented a framework for developing new exercises for epidemiologic computing. This system contained exercises on outbreak investigations, research survey, advanced Epi Info programming, and public health surveillance. The DoEpi exercises could be used in different curricula and for students of different background levels. Since then, a wide range of digital resources and online programs have emerged that are promoting epidemiology education across institutions and contexts.

These digital platforms may offer many advantages for the epidemiology learners, including flexible timing, avoiding unproductive time spent on commuting, spending more time and concentration on topics of interest, requiring fewer organizational resources that could be needed for in-person sessions, and facilitating interactions among fellow learners irrespective of their locations. However, these resources might come with some disadvantages, including some students may experience a lack of social and academic connectedness with peers and educators, some may find it difficult to adjust to the schedules for synchronous sessions, and 
most importantly, these systems shift most learning responsibilities to the learners who access and use the resources [14]. Educators and academic stakeholders should examine contextspecific benefits and challenges associated with using specific digital approaches for epidemiology education before adopting the same.

\section{Strengthening instructional and mentoring capacities}

Teaching epidemiology would require instructors who should have skills including but not limited to: i) sufficiently educated in epidemiologic methods and concepts, ii) competent in pedagogic approaches, iii) knowledgeable about the connection between epidemiologic theories and practice, and iv) can provide effective supervision for students with diverse backgrounds and academic interests. Alongside teaching competencies, it is necessary to emphasize individual mentoring in epidemiology and public health education [45, 46]. As discussed previously, students with varying knowledge and interests may require customized mentoring and supervision to learn and practice epidemiology, which would need additional support from the educators.

It is challenging to find caring mentors who are skilled in epidemiologic education. A degree in epidemiology or public health can be a bare minimum, whereas an efficient educator will have more to offer beyond her/his academic competencies. Being aware of the potential challenges faced by the learners would bring the educators closer to addressing those issues [21, 31]. In addition, proactive mentoring at the individual and group level may enhance educational outcomes in epidemiology. In the case of large classes, having multiple teaching assistants can improve the supervising process. For institutions and departments, periodic reviews of instructional resources and capacities may inform emerging challenges that should be prioritized to ensure optimal learning. Perhaps it is a common challenge for most institutions to bring and retain great minds in epidemiology, who can make the subject interesting to a diverse body of students and engage them in active learning processes. Perhaps, academic institutions should adopt a roadmap to develop intellectual pool of scholars who can advance local and regional capacities for epidemiologic education given the growing need for epidemiologists, health scientists, healthcare professionals, and epidemiology-literate citizens. 
Beyond traditional institutions of higher education and research, epidemiologic education is promoted across organizations and communities [3, 8, 47]. Many institutions support educators' education for strengthening school-level epidemiologic education [3, 4]. George and colleagues evaluated the outcomes of a workshop designed to prepare educators of middle and high school students [8]. This workshop introduced the basic concepts of epidemiology as well as a pedagogical framework for teaching the same. After the workshop, the majority (80\%) of the participants who taught epidemiology included epidemiologic concepts in other courses, and many of them perceived that learning epidemiology was a valuable experience for their students. Such capacity-building exercises for educators outside health science institutions may be helpful to promote epidemiology education in schools and communities, which may improve health literacy, behavior, practices, and outcomes in populations.

\section{Incorporating problem-solving practices in epidemiologic education}

There are many approaches for teaching epidemiology, including the use of textbooks, journal articles, and datasets $[9,14,22]$. However, educators increasingly recognize the role of problembased learning in epidemiologic education [48-50]. The learners may keep exploring different aspects of a public health problem from epidemiological perspectives and use epidemiologic methods to answer specific questions of interest. In this process, the educators can mentor them to make informed decisions during problem-solving. Such practices may reinforce the fundamental concepts and methods of epidemiology among the learners and empower them to use them in public health practice. Many scholars have explored such approaches for teaching epidemiology. For example, in a randomized controlled trial of traditional versus problem-based learning, Dyke and colleagues recruited 136 second-year medical students and enrolled them in a "problem-based learning course" or a traditional course [49]. Both groups of students took identical evaluations at the end of the program. After the trial, the problem-based learning group reported a better grasp of epidemiologic concepts, reported better satisfaction working with a group and became more enthusiastic about epidemiology practice-compared to the traditional course participants. In this study, non-consenting students who were enrolled in the traditional course were mostly of older age and from non-English speaking backgrounds, which reflect some barriers of deploying problem-based learning in teaching epidemiology. 
Different approaches have been proposed to promote problem-solving abilities through epidemiologic education and vice versa. Langmuir described the technique of using suspense through a "blind problem" where the educators may withhold the diagnosis of the disease until the end [50]. In this process, the learners are encouraged to confront the epidemiological data without being prejudiced by any preconceived notions about the epidemic or outbreak. Eventually, they may establish the source(s) of infection, mode(s) of spread, etiological factor(s), and examine specific control measures. Another problem-based approach was suggested by Garland and Pearson [48], who described the development of a course centered on the production of "research proposals" by the learners on their problems of choice. In this course, the lectures are combined with self-study resources for the learners. This problem-solving approach was proved both effective and more acceptable to students, and it improved learning outcomes as well as faculty-student interactions. Educators may explore innovative problem-solving approaches to teach epidemiology and facilitate the holistic development of their students.

\section{Experiential and simulation-based learning}

Some scholars suggested an advanced form of learning through gaining real-time experience of practicing epidemiologic methods and skills [18, 23, 37, 51]. Such experiential learning processes consider the complex nature of epidemiology, frequent loss of interest among learners in traditional lecturing, and the use of epidemiology in real life rather than memorizing the concepts. For example, Khapre and colleagues assessed the effectiveness of an experiential learning method in teaching epidemiology [51]. The module was prepared after orienting the facilitators and students to the new teaching methodology. The module was completed in 12 sessions, two hours per session. The students went through a series of 12 sessions (two hours for each session). At the end of the course, the learners exposed to experiential learning developed a higher interest in learning epidemiology and a high level of satisfaction than those exposed to lectures only. Epidemiology educators should seek potential opportunities to promote experiential learning. In such processes, they may establish partnerships with local health organizations and community groups where the learners can participate in epidemiologic research and practice, thus gaining invaluable experience beyond traditional class-based instructions. 
Another approach to facilitate experiential learning is organizing simulation-based events for teaching basic and applied epidemiology. Pandemic simulations, for example, often encourage the students to be aware of how the fundamentals of epidemiology can be used during a disease outbreak in populations. A mixed-methods study in an academic setting in rural Texas recruited 89 public health and nursing students to a simulation exercise that mirrored an influenza pandemic [52]. This study found an increased situational awareness [ $t=7.17(76), p<0.001]$, effective communication $[t=7.130(75), p<0.001]$, and public health knowledge $[t=8.77(74), p<$ 0.001] among the participants following the pandemic simulation. The findings of this study show potential of teaching applied epidemiology promoting experiential learning through simulation. Educators may include their concepts or methods of choice in simulation(s) and enable the students to learn or use the same as they participate in the simulation exercise(s).

\section{Team-based learning}

Mass lecturing and self-study are common learning processes across most academic disciplines. However, group-based collaborative learning is increasingly recognized as an effective teaching-learning approach in analytic disciplines [53,54]. Small groups of learners can work together, discuss critical concepts, provide feedback on individual or group perspectives, and address learning difficulties in epidemiology through interpersonal interactions. Moreover, group-based teaching is useful in developing skills on critical appraisal of qualitative and quantitative methodologies. In such approaches, the educators may pre-select papers and study resources and organize learners in multiple groups to evaluate those materials and discuss the same with their peers $[18,25,55]$. Moreover, team-based learning can be used through skilled learners who can prepare short presentations for their peers and engage them in discussions and mutual learning exercises.

Further, epidemiology educators may adopt "peer-mentoring" that is widely recognized as a teaching-learning strategy across scholarly disciplines [56-58]. In such exercises, the educators may identify advanced learners who may volunteer a group of students and support their intellectual development through structured activities and flexible interactive sessions. These processes may empower individuals and groups to engage meaningfully and practice knowledge-based leadership supporting collective learning and development [57, 58]. Moreover, 
peer-mentoring may save time and the need for expert mentoring if the misconceptions can be addressed at the group level, which would allow the learners to use the remaining time to learn more complex concepts and methods of epidemiology.

\section{Developing academic-practice partnerships}

Epidemiologic concepts and methods are often used in multidisciplinary public health research and practice $[19,55]$. Therefore, multidisciplinary collaborations can be useful to bring diverse perspectives for interpreting epidemiologic findings on a health condition or population $[25,37$, 59]. Also, emerging epidemiologists can offer great value to and benefit from multidisciplinary collaborations. From a professional viewpoint, the learners can build professional networks, develop confidence in clarifying learning outcomes, and gain quantitative research expertise on real-life examples. Also, engaging the learners in collaborative projects enables them to step beyond their known territories and prepares them to deal with academic and professional uncertainties.

Academic-practice partnerships can profoundly benefit individuals and institutions in many ways $[60,61]$. In such collaborations, the teaching academics can work with practitioners and industry partners to effectively bridge the teaching-research nexus in both directions. Specifically, in these ways, academic epidemiologists can make meaningful contributions to society by offering practice-based insights, building effective teaching programs using collaborative resources and strategies. For example, Novick and colleagues inform about an epidemiology teaching course developed at the University of Vermont's College of Medicine in collaboration with the staff members of the Vermont Department of Health [37]. This course focused on actual public health issues in Vermont. In the course's first year, 54\% of the students rated it as average or above average. Interestingly, $98 \%$ of the students in the second year rated their overall assessment as average or better, which suggested a remarkable success of this collaborative teaching program. This Vermont course communicated fundamental epidemiologic concepts emphasizing their clinical relevance in a recognizable community context using both public health and clinical perspectives. Such examples inform the academic benefits of collaborating with practice-based organizations for teaching epidemiology effectively. 


\section{Promoting continued and sustainable education}

Revisiting epidemiologic concepts and learning new developments in the subject may facilitate continued learning and application of updated measures among the practitioners. However, except for practicing epidemiologists, such exposures after completion of formal coursework may not be frequently available or accessible. Educators and institutions should consider the knowledge and practice gaps that may occur among the learners if there is a scarcity of such learning opportunities. Several strategies can be considered to bridge such gaps and empower the students beyond the scope of formal coursework. First, educators may introduce open access resources during formal academic sessions and encourage the learners to use the same for continued epidemiologic education $[14,62]$. Such resources, if organized and updated regularly, can connect both new and veteran learners and help them to keep learning epidemiology. Second, digital resources used during the coursework can be made accessible to the learners who registered once, which may be used as a refresher course if they want to revisit those contents $[11,14,19]$. Third, learning communities and institutions may introduce innovative learning strategies to keep the learners engaged in the field. For example, game-based or social media-based problem-solving exercises can be posted, and the subscribers can be asked to participate $[63,64]$. Such initiatives may reinforce fundamental concepts and methods among the learners and develop virtual communities who may wish to learn epidemiology together irrespective of their location or academic enrolment. Lastly, professional bodies should evaluate the scope of continued epidemiologic education within their broader academic goals and activities. Arguably, all professional institutions may benefit from integrating continued education sessions on epidemiology emphasizing health problems related to respective professions. Such efforts may encourage practicing health professionals to revisit classical epidemiology that can be applied in their professional capacities to improve evidence-based decision-making. These above-mentioned strategies may provide organized learning opportunities that are often not available after formal education, which would be critical for continued and applied learning in cost-effective ways. 


\section{Discussion}

This article reflected on the available evidence and opinions on teaching epidemiology. Several strategies and considerations were identified that may improve epidemiologic education in diverse contexts. These strategies and associated examples informed a wide range of techniques that may be useful for educators and institutions for addressing context-specific challenges related to teaching epidemiology. Prospective educational interventions and innovations should be adopted based on emerging evidence, best practices, and the preferences of the primary audience in teaching-learning exercises. However, the development and implementation of these educational measures may require a socio-ecological perspective, which may inform critical issues and opportunities.

Epidemiologic education can be organized for individuals who may interact with each other promoting interpersonal exchange and learning. Moreover, forums or groups of learners may facilitate knowledge transfer and sharing of skills within and between communities. These individual and collective learning may happen within or beyond formal institutions like schools or practice institutions. Irrespective of "places" where epidemiologic education may happen, the focus of the education remains at the community or population level, which makes most epidemiologic discourses fundamentally connected. For this reason, a broader and inclusive approach is needed to promote epidemiologic education in different frontiers of the society, including but not limited to educational institutions, health and social organizations, civil society, academic and professional institutions, and policymaking agencies. In different contexts, key stakeholders should consider local needs and resources, evaluate how they fit in broader epidemiologic and public health education, and adopt measures that ensure the continuum of education and practice in society.

Educators and institutions may benefit from the strategies and considerations outlined in this article. These measures can be adopted as stand-alone strategies on their own merits. However, they are likely to work best when implemented as a part of multiple strategies needed in unique educational contexts. To better understand what approaches would work effectively, educators may conduct situational analyses evaluating the intellectual needs of the learners and assess the efficacy of comparative strategies for specific problems of interest. Arguably, structured 
programs with little to no room for piloting different educational measures or modifying teaching approaches may affect the quality of epidemiologic educations. Perhaps, a combination of the best available evidence, use of acceptable theories, the inclusion of context-specific measures, and proactive engagement of the academic community may facilitate the advancement of epidemiologic education [9, 19, 21]. A multilevel well-coordinated approach may allow innovation and improve learning outcomes and make lasting impacts in public health literacy and informed health decision-making in institutions and societies.

\section{Limitations}

There are several limitations of this article that should be acknowledged before using the evidence, opinions, and insights presented here. First, this is a narrative review affected by the lack of a systematic methodology and the subjective perspectives of the author. Perhaps, a systematic and unbiased synthesis of empirical evidence could provide a more concrete view of existing literature. However, there is a scarcity of empirical studies on epidemiologic education. Therefore, this study calls for extensive primary research engaging students, educators, academic administrators, regulatory agencies, practice institutions, and communities to incorporate their views on how to teach and learn epidemiology. Moreover, most strategies presented in this article can be useful for teaching other topics under the broad umbrella of public health, which makes these strategies less specific for epidemiologic education. Further research is needed to specify the transdisciplinary approaches and unique measures useful for teaching and learning epidemiology. Furthermore, articles included in this review provide limited guidance on how to organize resources and policies for the effective implementation of these strategies. Scholars and practitioners should reflect on implementation challenges and opportunities for effective epidemiologic education in different settings and facilitate widespread communication for knowledge exchange and translation. Lastly, the role of institutional, national, and global stakeholders on epidemiologic education is not clearly discussed in the current literature, which appears to be a knowledge gap with profound implications for policymaking and future agenda-setting. Those key stakeholders should initiate extensive collaborations and develop a shared pool of resources for promoting epidemiologic 
education considering the transdisciplinary application of epidemiologic concepts and methods. Further implementation research and collaborative educational efforts should address the limitations of this review and advance epidemiologic education globally.

\section{Conclusions}

Epidemiologic teaching is critical for effective learning and subsequent applications of epidemiologic concepts and methods in public health professionals and other groups of learners with diverse backgrounds and interests. Existing strategies and approaches may help the educators and learning communities to find what might work in respective contexts. Also, educational innovations should be encouraged and widely communicated, considering the scarcity of implementation research in this area. Lastly, future efforts to promote epidemiologic and public health education must be inclusive and evidence-based, which may bridge the existing and emerging gaps in teaching-learning discourses.

\section{Acknowledgment: None}

Funding information: No funding or financial support was received for preparing this manuscript. Conflicts of interest: None. 


\section{References}

[1] Frérot M, Lefebvre A, Aho S, et al. What is epidemiology? Changing definitions of epidemiology 1978-2017. PLoS One; 13. Epub ahead of print 1 December 2018. DOI: 10.1371/JOURNAL.PONE.0208442.

[2] Lauer MS. The Basic Science That is Epidemiology. JACC Cardiovasc Imaging 2014; 7: 879.

[3] Kaelin MA, Huebner WW, Cordell RL, et al. Professional Development for Prospective Epidemiology Teachers in Grades 6-12. Public Health Rep 2008; 123: 5.

[4] D'Agostino EM. High School Epidemiology: Helping Students Practice Science Through Guided Inquiry and Authentic Learning Strategies. Pedagog Heal Promot 2019; 5: 293-298.

[5] D'Agostino E. Public Health Education: Teaching Epidemiology in High School Classrooms. Am J Public Health 2018; 108: 324.

[6] Medicine I of. Who Will Keep the Public Healthy?: Educating Public Health Professionals for the 21st Century. Who Will Keep Public Heal. Epub ahead of print 4 November 2002. DOI: $10.17226 / 10542$.

[7] Cordeira KL, Cordell R. Epidemiology and Public Health Science: Core Competencies for High School Students.

[8] St. George DMM, Chukhina M, Kaelin MA. Training Teachers to Teach Epidemiology in Middle and High Schools. Int Q Community Health Educ 2017; 38: 65-69.

[9] Keyes KM, Galea S. Current practices in teaching introductory epidemiology: How we got here, where to go. Am J Epidemiol 2014; 180: 661-668.

[10] Marantz PR. Epidemiology 101. Toward an Educated Citizenry. Am J Prev Med 2008; 35: 264268.

[11] Werler MM, Stuver SO, Healey MA, et al. The Future of Teaching Epidemiology. Am J Epidemiol 2019; 188: 825-829.

[12] Berglund A. What's in a Word? Concept mapping: A graphical tool to reinforce learning of epidemiological concepts. J Epidemiol Community Health 2015; 69: 1232-1236.

[13] Abraham A, Gille D, Puhan MA, et al. Defining Core Competencies for Epidemiologists in Academic Settings to Tackle Tomorrow's Health Research Challenges: A Structured, Multinational Effort. Am J Epidemiol 2021; 190: 343-352. 
[14] Caron RM. Teaching epidemiology in the digital age: Considerations for academicians and their students. Ann Epidemiol 2013; 23: 576-579.

[15] Grabowski B. "P < 0.05" Might Not Mean What You Think: American Statistical Association Clarifies P Values. JNCI J Natl Cancer Inst; 108. Epub ahead of print 1 August 2016. DOI: 10.1093/JNCI/DJW194.

[16] Burns CJ, Wright JM, Pierson JB, et al. Evaluating Uncertainty to Strengthen Epidemiologic Data for Use in Human Health Risk Assessments. Environ Health Perspect 2014; 122: 1160.

[17] Robertson JF. Teaching Epidemiology. Nurse Educ 2002; 27: 123-125.

[18] Marantz PR, Croen L, Wassertheil-Smoller S, et al. Teaching clinical epidemiology to medical students using a collaborative learning model. Am J Prev Med 1991; 7: 121-123.

[19] Banack HR, Lesko CR, Whitcomb BC, et al. Teaching Epidemiology Online (Pandemic Edition). Am J Epidemiol 2021; 190: 1183-1189.

[20] Jenicek M, Fletcher RH. Epidemiology for Canadian medical students - desirable attitudes, knowledge and skills. Int J Epidemiol 1977; 6: 69-72.

[21] Moffat M, Sinclair HK, Cleland JA, et al. Epidemiology teaching: Student and tutor perceptions. Med Teach 2004; 26: 691-695.

[22] Romm FJ, Dignan M, Herman JM. Teaching clinical epidemiology: a controlled trial of two methods. Am J Prev Med 1989; 5: 50-51.

[23] Huang C, Hsieh J, Sun C, et al. Teaching Epidemic Simulation and Policymaking to Novice Researchers. 2006; 2006: 393-398.

[24] James EL, Graham ML, Snow PC, et al. Undergraduate Students in the Health Sciences. 2006; 575-578.

[25] James EL, Graham ML, Snow PC, et al. Teaching research and epidemiology to undergraduate students in the health sciences. Aust N Z J Public Health 2006; 30: 575-578.

[26] Ali KF. Public Health Education through the Lens of Social Media: Implications in the COVID-19 Era. Sultan Qaboos Univ Med J 2021; 21: e143.

[27] van den Broucke S. Why health promotion matters to the COVID-19 pandemic, and vice versa. Health Promot Int 2021; 35: 181-186.

[28] Nelson-Hurwitz DC, Tagorda M, Kehl L, et al. Developing an Undergraduate Public Health Introductory Core Course Series. Front Public Heal 2018; 6: 155. 
[29] White F. The Imperative of Public Health Education: A Global Perspective. Med Princ Pract 2013; 22: 515.

[30] Moseley D, Baumfield V, Elliott J, et al. Frameworks for thinking : a handbook for teaching and learning. Cambridge Cambridge Univ Press. Epub ahead of print 2005. DOI:

$10.2277 / 0521848318$.

[31] Shir N, Bidabadi A, Isfahani AN, et al. Effective Teaching Methods in Higher Education: Requirements and Barriers. J Adv Med Educ Prof 2016; 4: 170.

[32] Avelino-silva V, Zablotska L, Martin J. 591The "Big 6": A purpose-based framework for motivating and teaching epidemiologic methods. Int J Epidemiol 2021; 50: 2021.

[33] Jensen DH, Jetten J. Bridging and bonding interactions in higher education: social capital and students' academic and professional identity formation. Front Psychol; 6. Epub ahead of print 2015. DOI: 10.3389/FPSYG.2015.00126.

[34] Bourdieu P. The Forms of Capital. Sociol Econ Life 2018; 78-92.

[35] Rabbani F, Shipton L, White F, et al. Schools of public health in low and middle-income countries: An imperative investment for improving the health of populations? BMC Public Health 2016; 16: 1-12.

[36] Shy CM, Walker AM. The failure of academic epidemiology: witness for the prosecution. Am J Epidemiol; 145. Epub ahead of print 1997. DOI: 10.1093/OXFORDJOURNALS.AJE.A009133.

[37] Novick LF, Greene C, Vogt RL. Teaching medical students epidemiology: Utilizing a State Health Department. Public Health Rep 1985; 100: 401-405.

[38] Epidemiology in the Classroom | Teacher Roadmap | Career Paths to Public Health |CDC, https://www.cdc.gov/careerpaths/k12teacherroadmap/classroom/index.html (accessed 17 January 2022).

[39] Cheng SO, Liu A. Using online medical education beyond the COVID-19 pandemic - A commentary on "The coronavirus (COVID-19) pandemic: Adaptations in medical education ". Int J Surg 2020; 84: 159.

[40] Pei L, Wu H. Does online learning work better than offline learning in undergraduate medical education? A systematic review and meta-analysis. Med Educ Online; 24. Epub ahead of print 1 January 2019. DOI: 10.1080/10872981.2019.1666538.

[41] Martin F, Sun T, Westine CD. A systematic review of research on online teaching and learning from 2009 to 2018. Comput Educ 2020; 159: 104009. 
[42] Mobasheri M, Kheiri S, Mardanpour E, et al. Effects of epidemiology learning software on nursing and midwifery students. Med I Islam Repub Iran 2014; 28: 1-5.

[43] Russell KM, Miller AM, Czerwinska J. Epidemiology for community health nursing: an interactive computer assisted instruction program. Comput Nurs 1994; 12: 98-105.

[44] Dean AG, Shah SP, Churchill JE. DoEpi: Computer-assisted instruction in epidemiology and computing and a framework for creating new exercises. Am J Prev Med 1998; 14: 367-371.

[45] Mahayosnand PP, Stigler MH. The need for mentoring in public health. AmJ Public Health 1999; 89: 1262.

[46] Burgess A, van Diggele C, Mellis C. Mentorship in the health professions: a review. Clin Teach 2018; 15: 197-202.

[47] Kaelin MA, Huebner WW. Epidemiology, health literacy, and health education. Am J Heal Educ 2002; 33: 362-364.

[48] Garland BK, Pearson RJC. Epidemiology Course for Medical Students Focuses on Proposal Writing. Am J Prev Med 1989; 5: 240-243.

[49] Dyke P, Jamrozik K, Plant AJ. A randomized trial of a problem-based learning approach for teaching epidemiology. Acad Med 2001; 76: 373-379.

[50] Langmuir AD. The use of the 'blind' problem in teaching epidemiology. Int J Epidemiol 1975; 4: 253-256.

[51] Khapre M, Gupta M, Kishore S. Experiential Learning in Epidemiology for Medical Undergraduates: A MixedMethod Approach. J Clin Diagnostic Res. Epub ahead of print 2019. DOI: 10.7860/jcdr/2019/38297.12488.

[52] Gandhi S, Yeager J, Glaman R. Implementation and evaluation of a pandemic simulation exercise among undergraduate public health and nursing students: A mixed-methods study. Nurse Educ Today 2021; 98: 104654.

[53] Strijbos JW, Martens RL, Jochems WMG. Designing for interaction: Six steps to designing computer-supported group-based learning. Comput Educ 2004; 42: 403-424.

[54] Laal M, Laal M. Collaborative learning: what is it? Procedia - Soc Behav Sci 2012; 31: 491-495.

[55] Abramson JH. Teaching epidemiology inside and outside the classroom. Public Health Rev 2002; 30: 339-363. 
[56] Hall R, Jaugietis Z. Developing Peer Mentoring through Evaluation. Innov High Educ 2011; 36: 41-52.

[57] Colvin JW, Ashman M. Roles, Risks, and Benefits of Peer Mentoring Relationships in Higher Education. http://dx.doi.org/101080/13611261003678879 2010; 18: 121-134.

[58] Leidenfrost B, Strassnig B, Schütz M, et al. The Impact of Peer Mentoring on Mentee Academic Performance: Is Any Mentoring Style Better than No Mentoring at All?. Int J Teach Learn High Educ 2014; 26: 102-111.

[59] Chih H, Firth A. 1069Reflection from working with multidisciplinarymultidisciplinary teams on practice of teaching epidemiology. Int J Epidemiol 2021; 50: 2021.

[60] Hall ZW, Scott C. University-Industry Partnership. Science (80- ) 2001; 291: 553-553.

[61] Wong JM, Bielak L, Eddy RG, et al. An Academia-Industry Partnership for Planning and Executing a Community-Based Feeding Study. Curr Dev Nutr; 2. Epub ahead of print 1 September 2018. DOI: 10.1093/CDN/NZY060.

[62] Clinical Epidemiology (MOOC) - Continuing Education - Utrecht University, https://www.uu.nl/en/professionals/programmes/clinical-epidemiology-mooc (accessed 17 January 2022).

[63] Bauman EB. Game-based teaching and simulation in nursing and healthcare. 265.

[64] Hardy S, Dutz T, Wiemeyer J, et al. Framework for personalized and adaptive game-based training programs in health sport. Multimed Tools Appl 2015; 74: 5289-5311. 